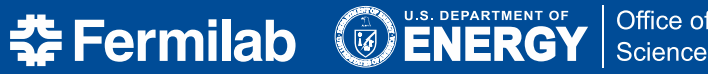

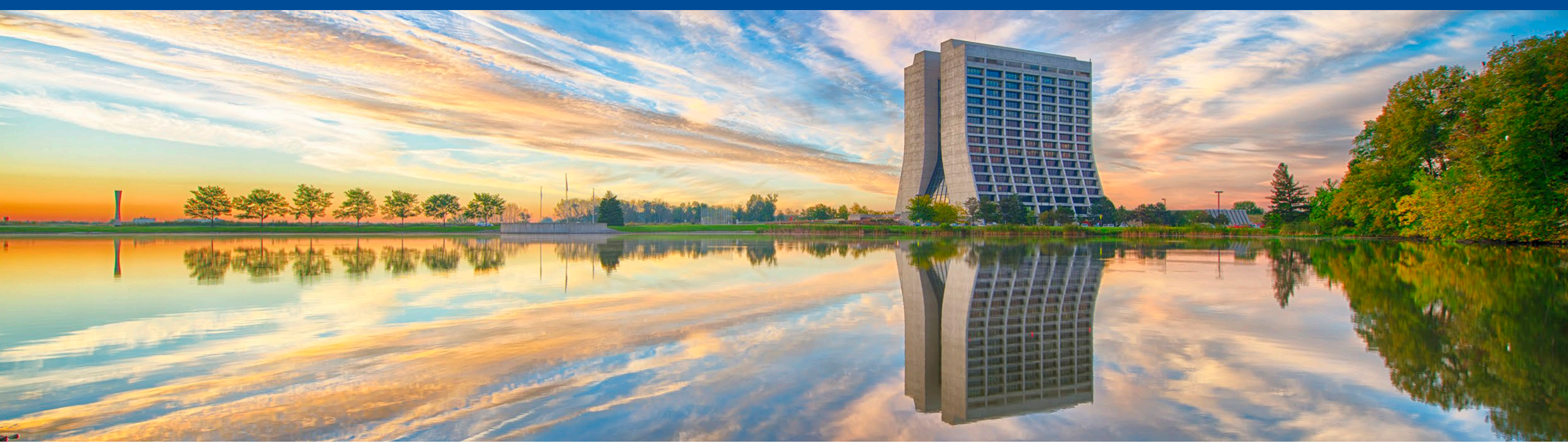

\section{Streamlining FIMS/CAIS Data Validation with GIS}

Dawn McWha, GIS Manager, Fermi National Accelerator Lab DOE GIS User Group Meeting, Esri Federal User Conference, Washington, D.C. 29 January 2019

This manuscript has been authored by Fermi Research Alliance, LLC under Contract No. DE-AC02-07CH11359 with the U.S. Department of Energy, Office of Science, Office of High Energy Physics. 


\section{Today's Topics}

- Orientation to Fermilab; GIS program

- Fermilab Infrastructure Database (FID)

- Fermilab Location Data Standards

- Space Utilization and Management (FIMS Reporting)

- FIMS Source Documentation

- Condition Assessments 


\section{Fermi National Accelerator Laboratory (Fermilab/S}

- Structures

- 460 Properties

- 402 buildings (367) and trailers (36) (2.4 million sq. ft.)

- 537 floors

- 144 tornado shelter designations

- 54 OSFs

- Underground enclosures and tunnels (1.1 million sq. ft.)

- 3 lands

- Land Features

- 36 miles of roads; 130 acres of parking lots

- 15 major waterbodies (13.7 million sq. ft.); 16 miles of streams

- 146 acres of highly sensitive ecological landscape; lots of wildlife

- Utilities

- 115 miles of electric cable; 2 primary substations; 241 secondary s

- 27 miles of industrial cooling water; fire protection and science

- 19 miles of domestic water; 18 miles of natural gas pipeline; 14 mil

- $1,750+$ employees; visiting scientists

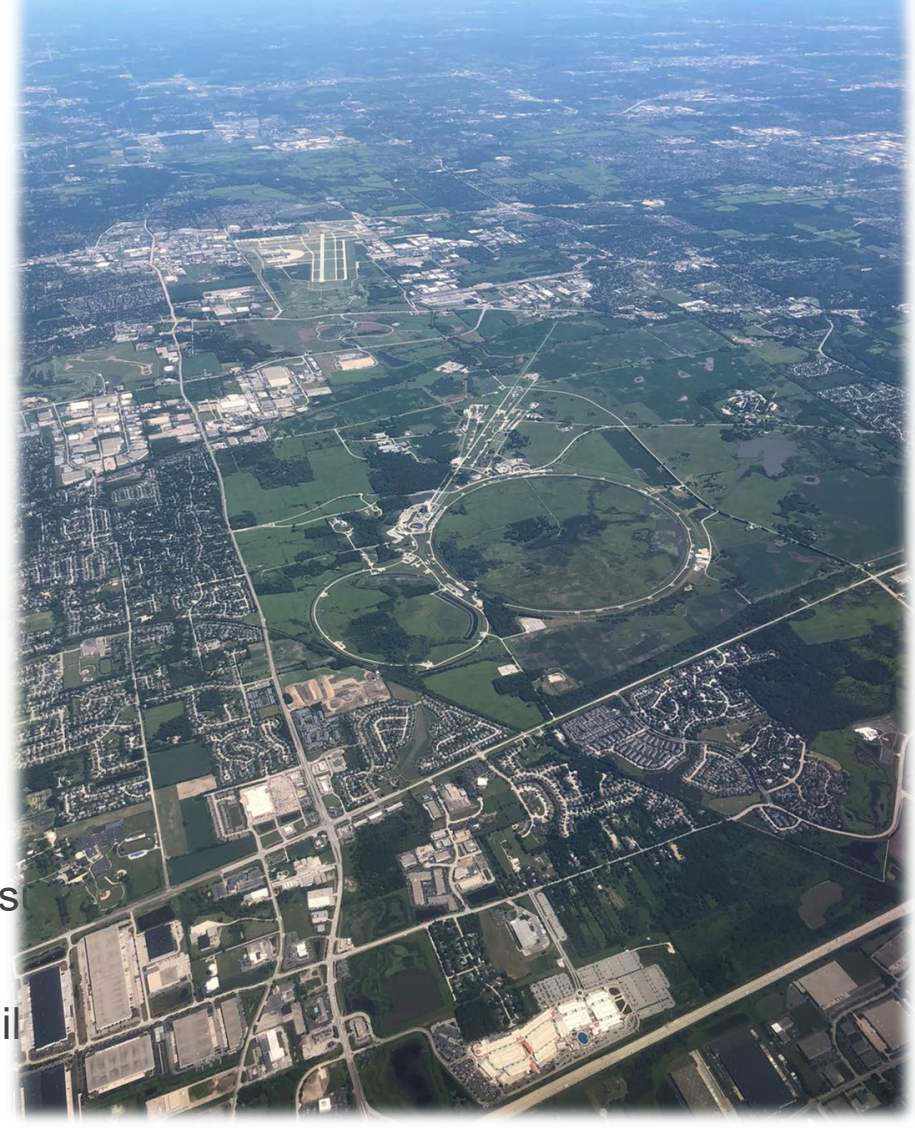

D. McWha | Fermilab | 2019 Esri Federal User Conference | USDOE GIS User Group Meeting 


\section{Fermilab GIS Program}

- The Fermilab GIS Program is within the Site Services Department of Facilities Engineering Services Section (FESS) under the Office of the Chief Operating Officer.

- FESS contains the Engineering, Facilities Management, Logistics and Property Control and Site Services Departments (SSV).

- The Data Services group within SSV programmatically assumes all lab-wide Real Property (1), GIS (1) and Application and Data functions (1).

- Our GIS supports all Divisions and Sections with their GIS needs. 


\section{Real Property}

- Real property includes land and

\section{U.S. Department of Energy} anything permanently affixed to it.

Facilities Information Management System

- Buildings, Trailers, Other Structures and Facilities (OSF's) such as roads and utility systems.

- DOE Order 430.1C Real Property Asset Management order

- Requires the Department of Energy to keep track of all of their real property assets in a database called the Facilities Information Management System (FIMS).

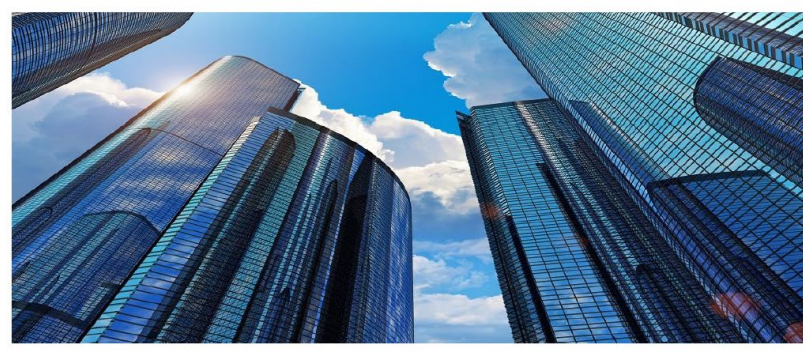




\section{Fermilab Infrastructure Database (FID)}

- FID is Fermilab's in-house facility infrastructure database used to manage real property data.

- FID has been in place at Fermilab since 2011 and is continually being enhanced.

- FID ...

- Populates FIMS - data from FID is uploaded into FIMS

- Provides a single place allowing Property Managers to easily review, access, validate, and export validate data

- Eliminates redundant, stale data; no more Excel spreadsheets

- Supports the annual FIMS Validation

- Declared a "Best Practice" by OAPM

- Integrates with GIS and other information stores (CMMS, EAM, Engineering) 


\section{GIS and FID in Action}

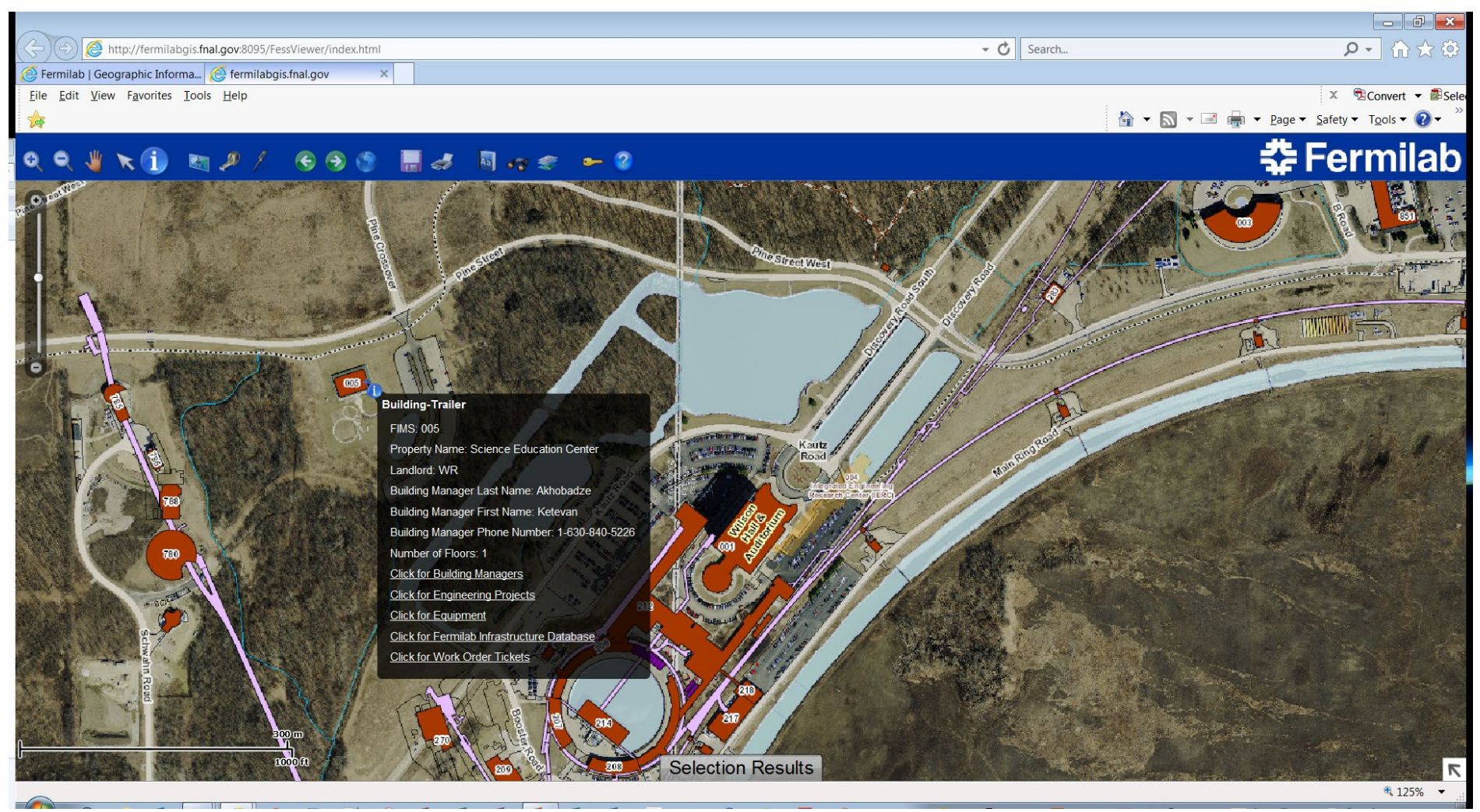




\section{GIS and FID in Action}

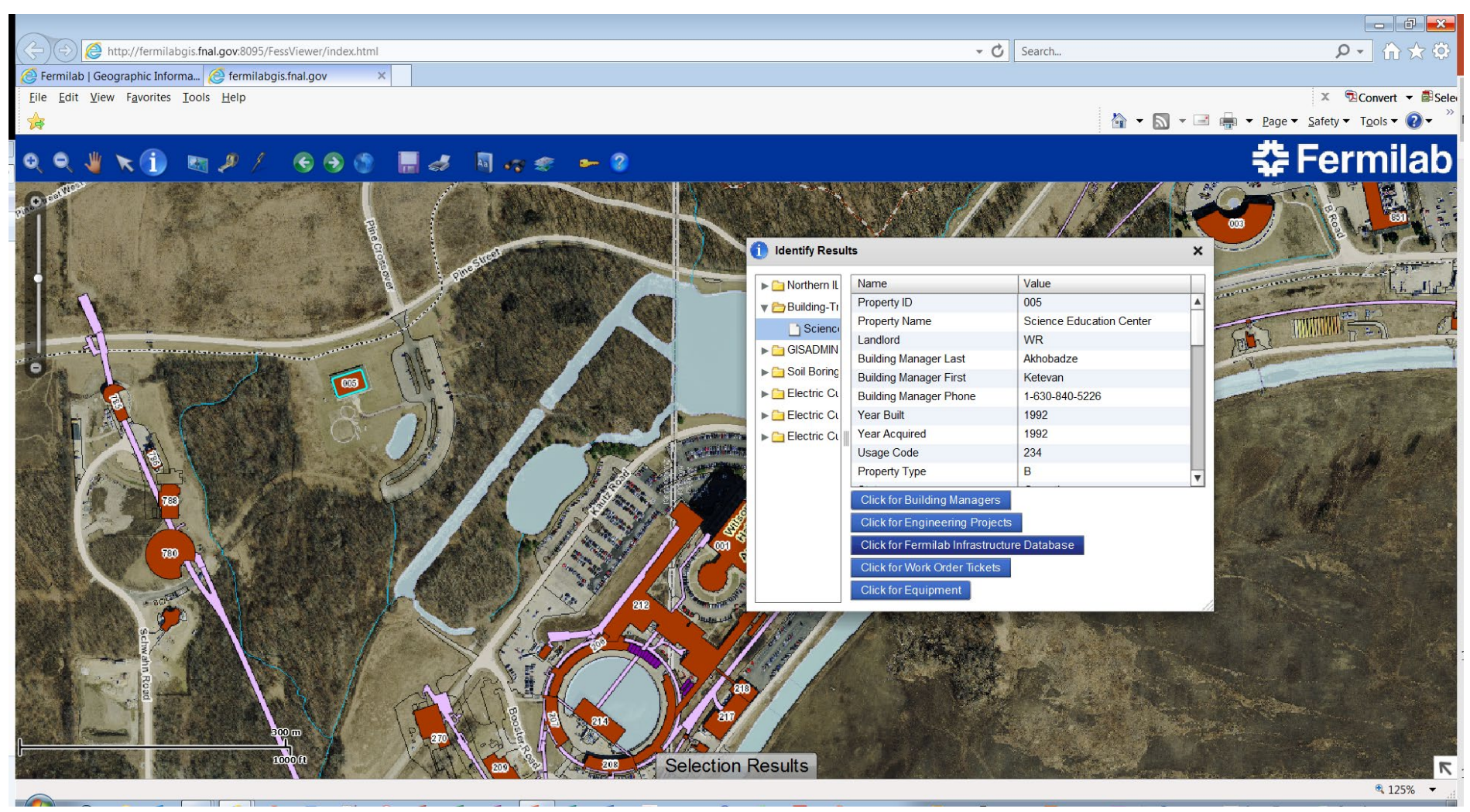




\section{GIS and FID in Action}

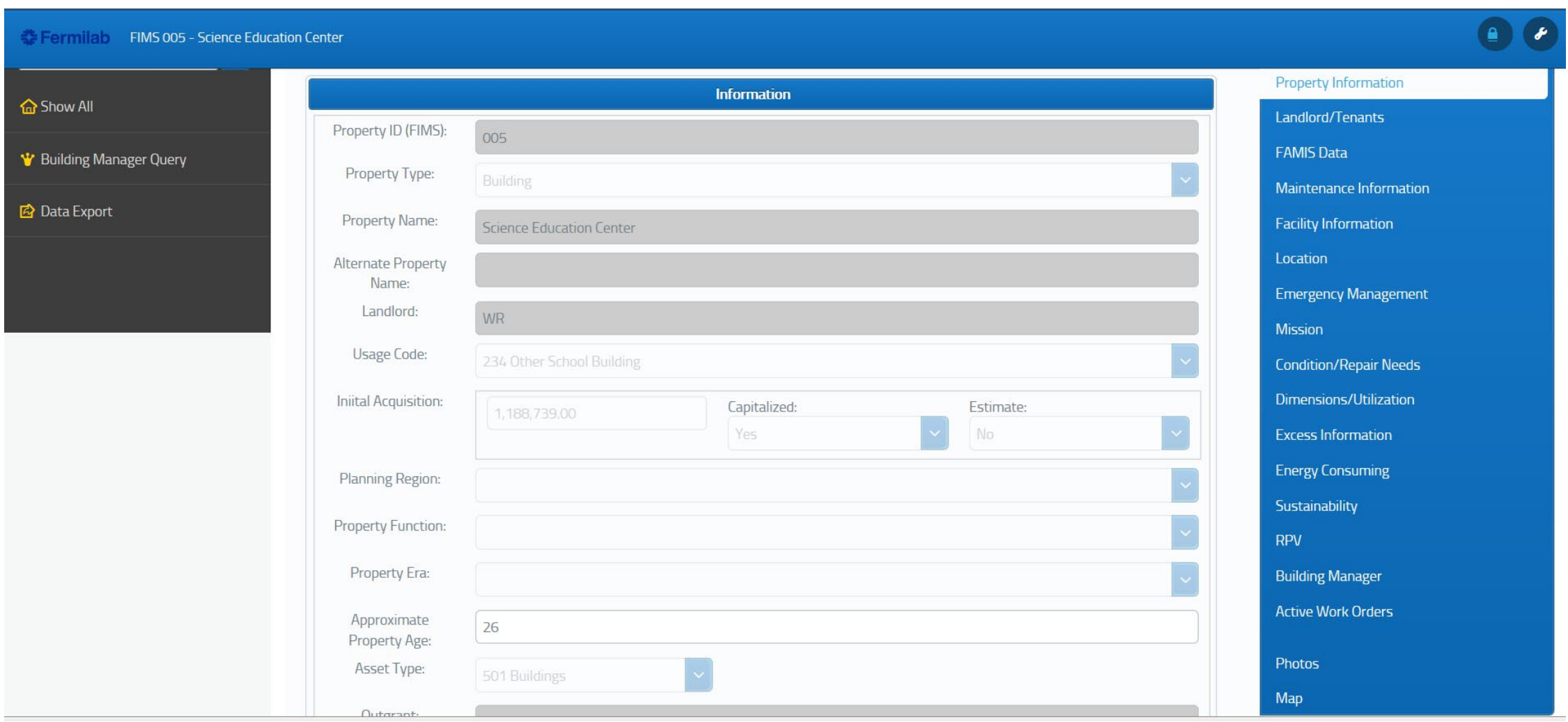




\section{Facility - By Mission Dependency}

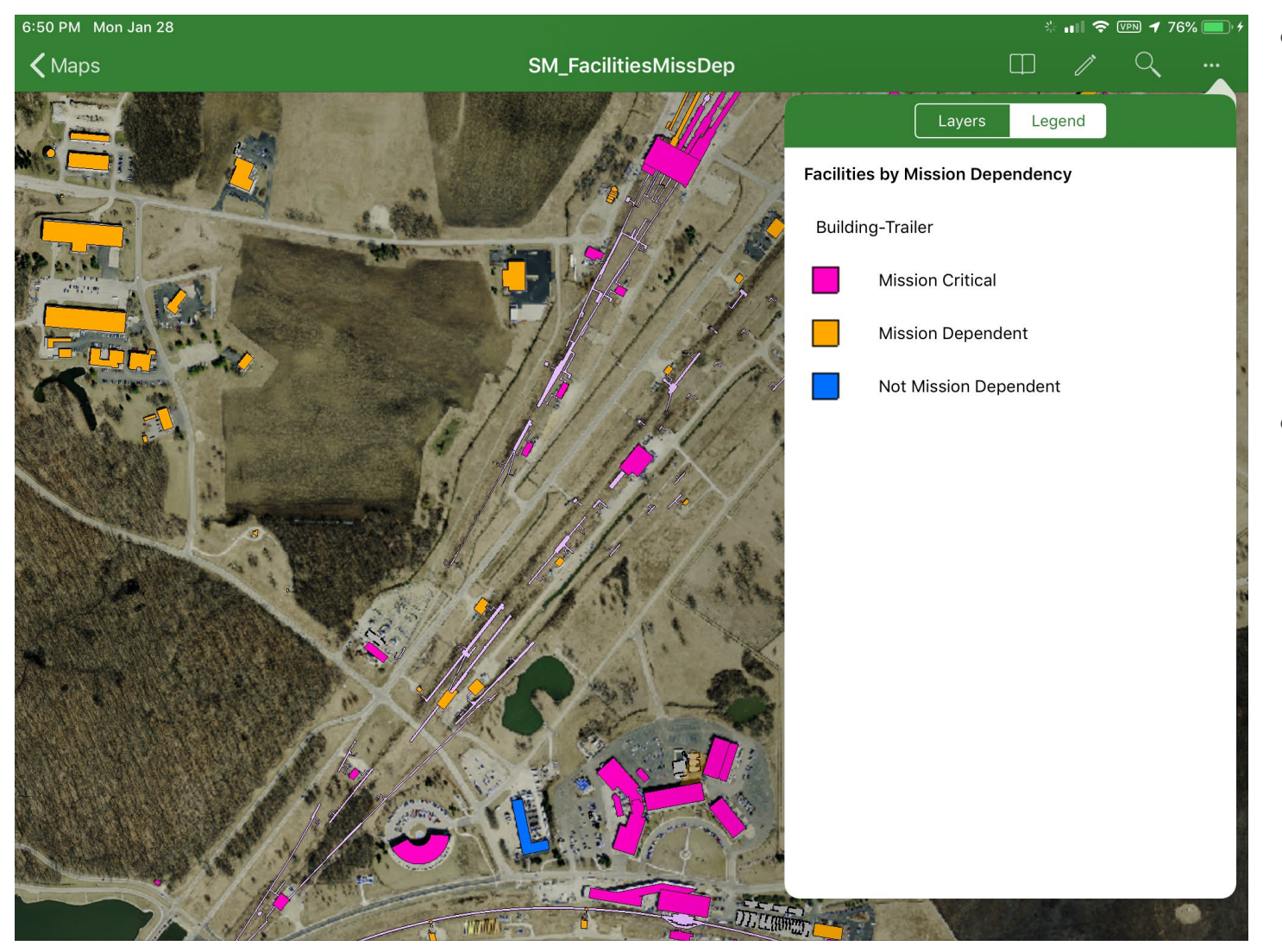

D. McWha | Fermilab | 2019 Esri Federal User Conference | USDOE GIS User Group Meeting
- Mission Dependency

- Visualize

- Query

- Analyze

- Count

- Mission Critical (122)

- Mission Dependent (243)

- Not Mission Dependent (37) 


\section{Facility - By Usage}

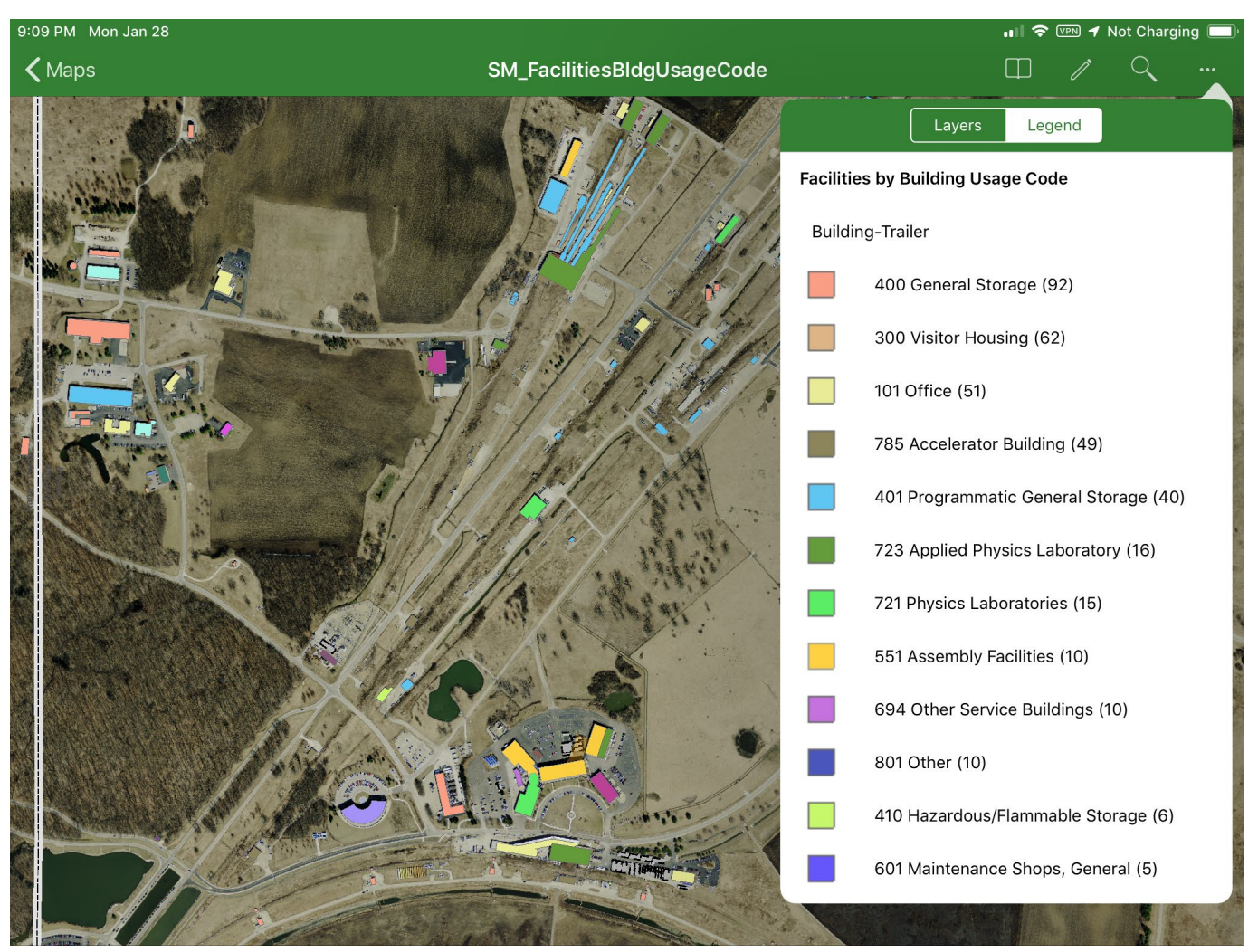

- Usage Code

- Visualize

- Query

- Analyze

- Fermilab currently has 33 unique Usage Codes 


\section{Fermilab Location Data Standards}

- 2017 - Location Data Standards Task Force established

- Establish consistent naming, conventions and definitions for location data

- Establish rules on their assignment through the Fermilab Facility Lifecycle (early design through operation and decommissioning)

- Location Data - is data content associated with the geographic location of real property (land, building, trailer or other structures and facilities (OSF).

- Location Data Standards - uniform and consistent naming, grouping, nomenclature, definition, terminology and applicability of the Location Data content.

- Applied throughout the lifecycle of all planned and in place real property assets located on and associated with Fermilab.

- GIS is the system of record of all location data; feeds other systems 


\section{Fermilab Location Data Standards}

\begin{tabular}{|c|c|c|}
\hline Group & Location Data Standard & Members/Scope \\
\hline Political & $\begin{array}{l}\text { Country, State, County, Township, } \\
\text { Municipality, Zipcode }\end{array}$ & All Real Property \\
\hline Agency & Agency, Program, Site, Area & All Real Property \\
\hline Area & Quadrant, Campus, Complex, Property & All Real Property \\
\hline Property & $\begin{array}{l}\text { Property ID, Property Name, Property Address, } \\
\text { Property USNG Coordinate }\end{array}$ & All Real Property \\
\hline Facility & Floor and Room & Building, Trailer and Enclosure Real Property \\
\hline \multirow[t]{3}{*}{ Space } & Unoccupied Space (As Designed) & \multirow[t]{3}{*}{ Building, Trailer and Enclosure Real Property } \\
\hline & Unoccupied Space (In Place) & \\
\hline & Occupied Space (In Place) & \\
\hline
\end{tabular}




\section{Fermilab Location Data Standards - GIS Layers}

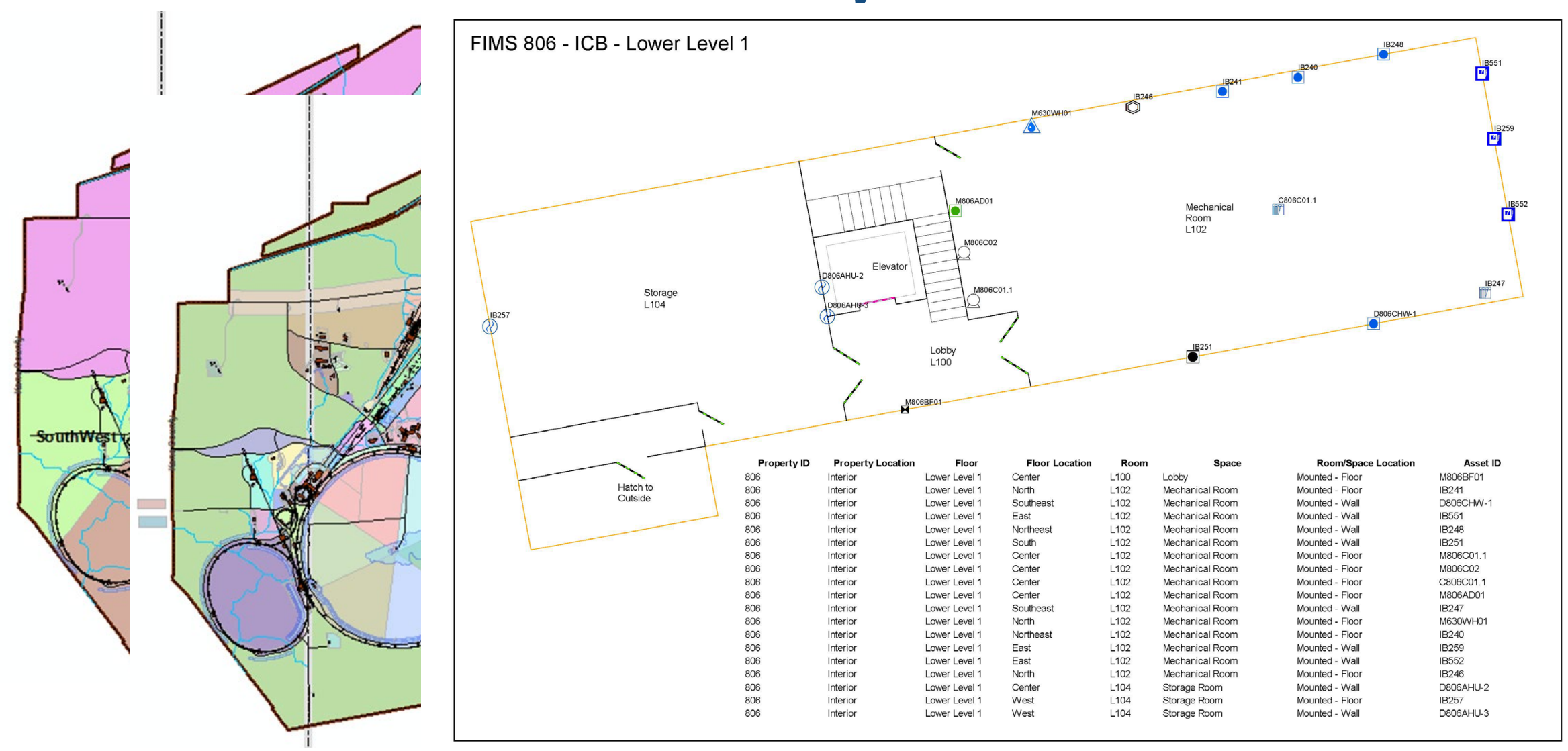




\section{Location Data Standards - Space}

- AISLE

- ALCOVE

- ANALOg ROOM

- ATTIC

- BATHROOM

- BEAM

- BEDROOM

- BREAKROOM

- BRIDGE

- CAFÉ

- CATWALK

- CHASE

- CHUTE

- circulation aREA

- column

- CLASSROOM (LAB)

- CLASSROOM (LECTURE)

- CLEANROOM

- CLOSET

- COLUMN
- COMMON AREA

- COMMUNICATION ROOM

- COMPUTER ROOM

- cONFERENCE ROOM

- CORRIDOR

- COUNTING ROOM

- DINING AREA

- DINING ROOM

- DISPLAY AREA

- DUMBWAITER

- ELECTRICAL ROOM

- ELEVATOR

- ENTRY (LOBBY)

- ENTRY (VESTIBULE)

- EQUIPMENT ROOM

- ESCALATOR

- garage

- gaRdEN

- HALLWAY

- hallway (OFFICE)
- HIGH BAY

- JANITOR ROOM

- KITCHEN

- LAB (MECHANICAL)

- LAB (SCIENCE)

- LAUNDRY

- library

- LIVING ROOM

- LOBBY

- LOCKER ROOM

- MATERIAL PROCESSING AREA

- MECHANICAL ROOM

- OFFICE

- OFFICE (HOME)

- PARKING

- PIT

- PLATFORM

- PORCH

- QUIET ROOM

- RECROOM
- RESTROOM

- ROOFOCCU

- ROOFUNOC

- SHAFT

- SHELTER

- SHOP (MACHINE)

- SHOP (METAL)

- SHOP (WOODWORKING)

- SHOP (WORK)

- STAIRWELL

- STORAGE

- STORAGE (OFFICE)

- technical aREA

- THEATER

- travelator

- TUNNEL

- UTILITY ROOM

- VAULT

- WALKWAY 


\section{FIMS Validation Requirement - Facility Dimensions/Space Utilization}

- Gross and Usable (Net) Square Footage

- Space (Function) cross-walked to "Space Types"

- General - Dry

- General - Wet

- High Bay

- Office

- Power Intensive

- Ventilation Intensive

- Storage

- "Office" (2018); "Storage" (2019)

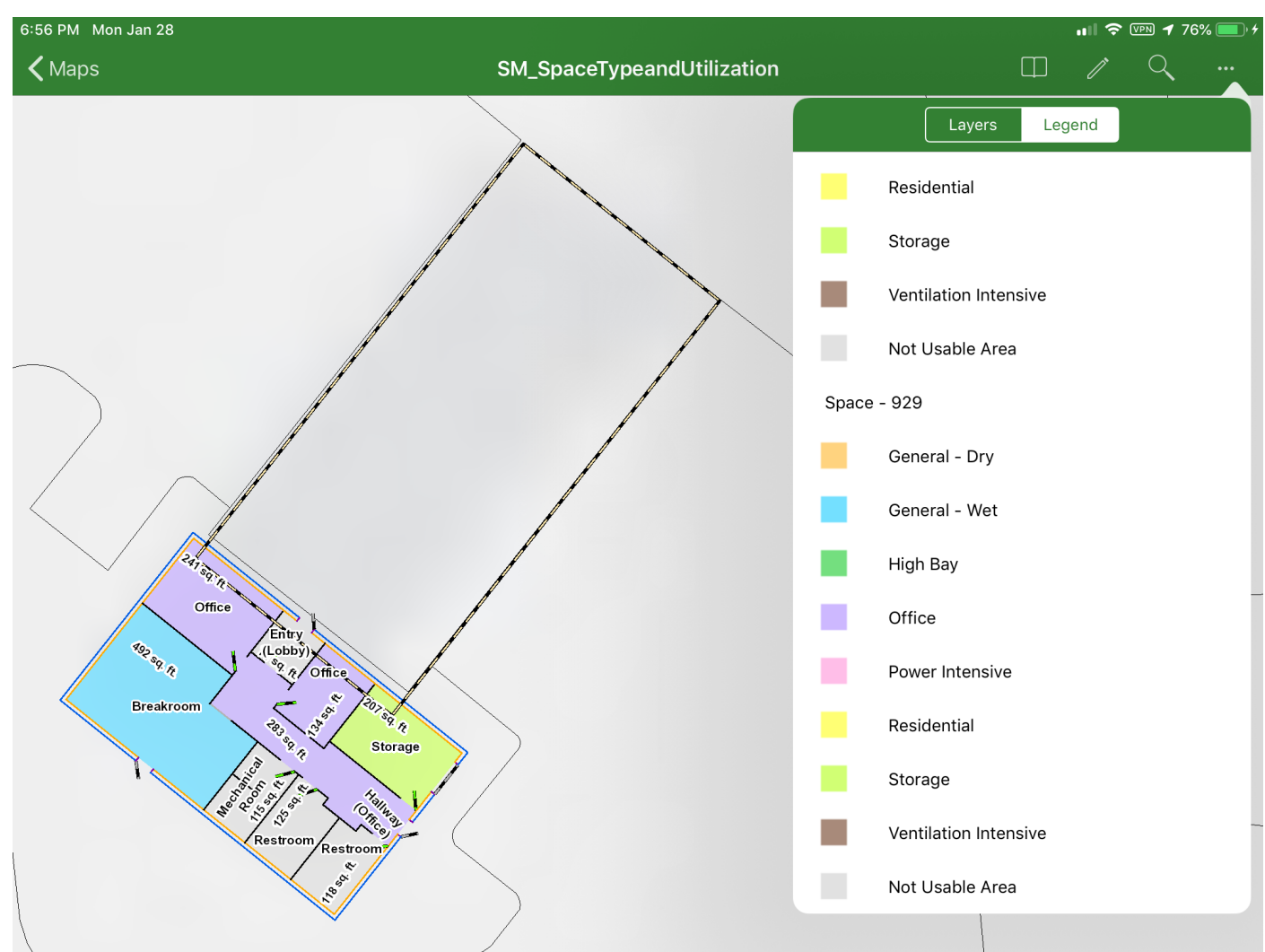




\section{Source Documentation (Past)}

\section{Gross SF:}

Usable Squtt

No of Floors:

\%Utilized

Utilization Level

Asset:
Space
Type:

Asset: $\quad 100 \%$

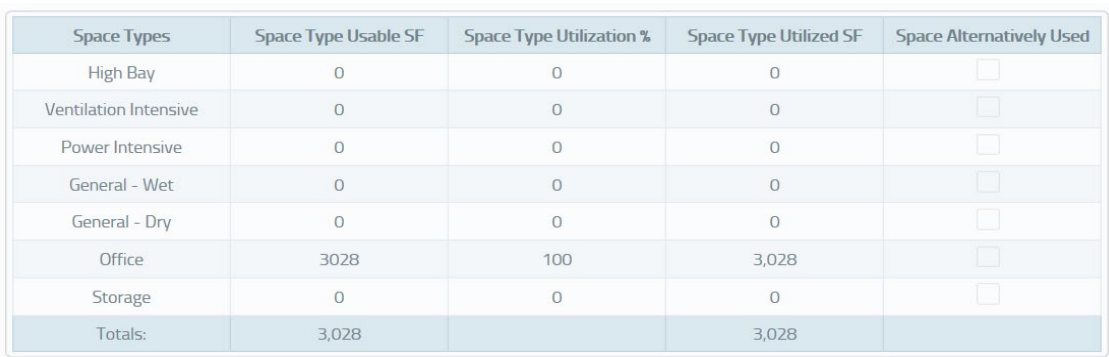

BUILDING DATA REQUEST FORI

REAL PROPERTY PHYSICAL INVENTORY - 1993

BUILDING:BUILDING MANAGER DATS

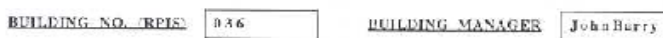

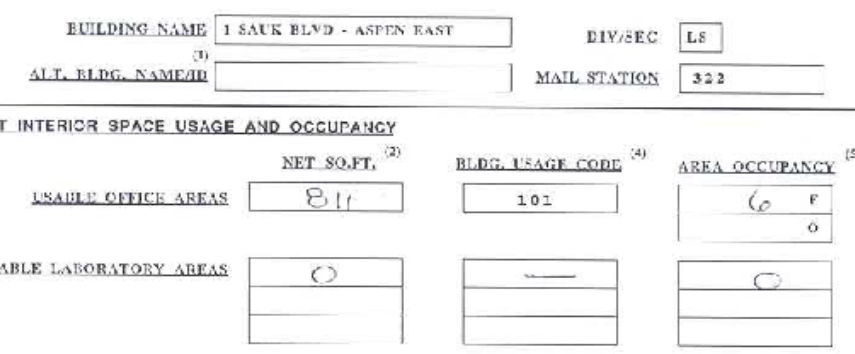

OTICR USAMIIT AFE:S
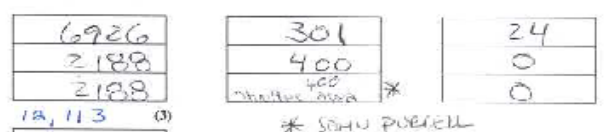

SON-USABLE. SBESS 2,113 * Sontu puerell

\begin{tabular}{|c|c|}
\hline \multicolumn{2}{|c|}{ 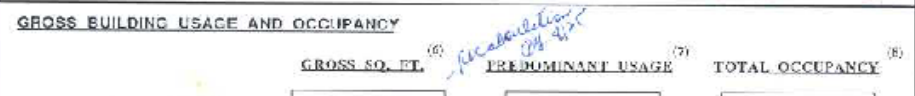 } \\
\hline$\frac{t+167}{10110}$ & \begin{tabular}{|l|l|}
301 & 30 \\
\end{tabular} \\
\hline 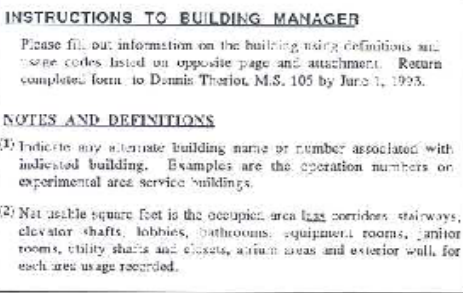 & 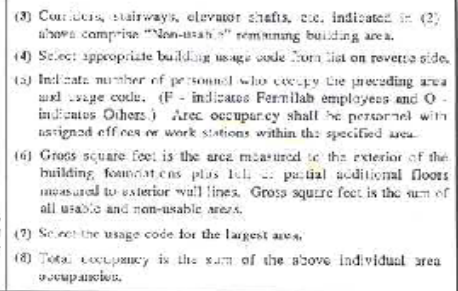 \\
\hline
\end{tabular}




\section{Source Documentation (Past)}
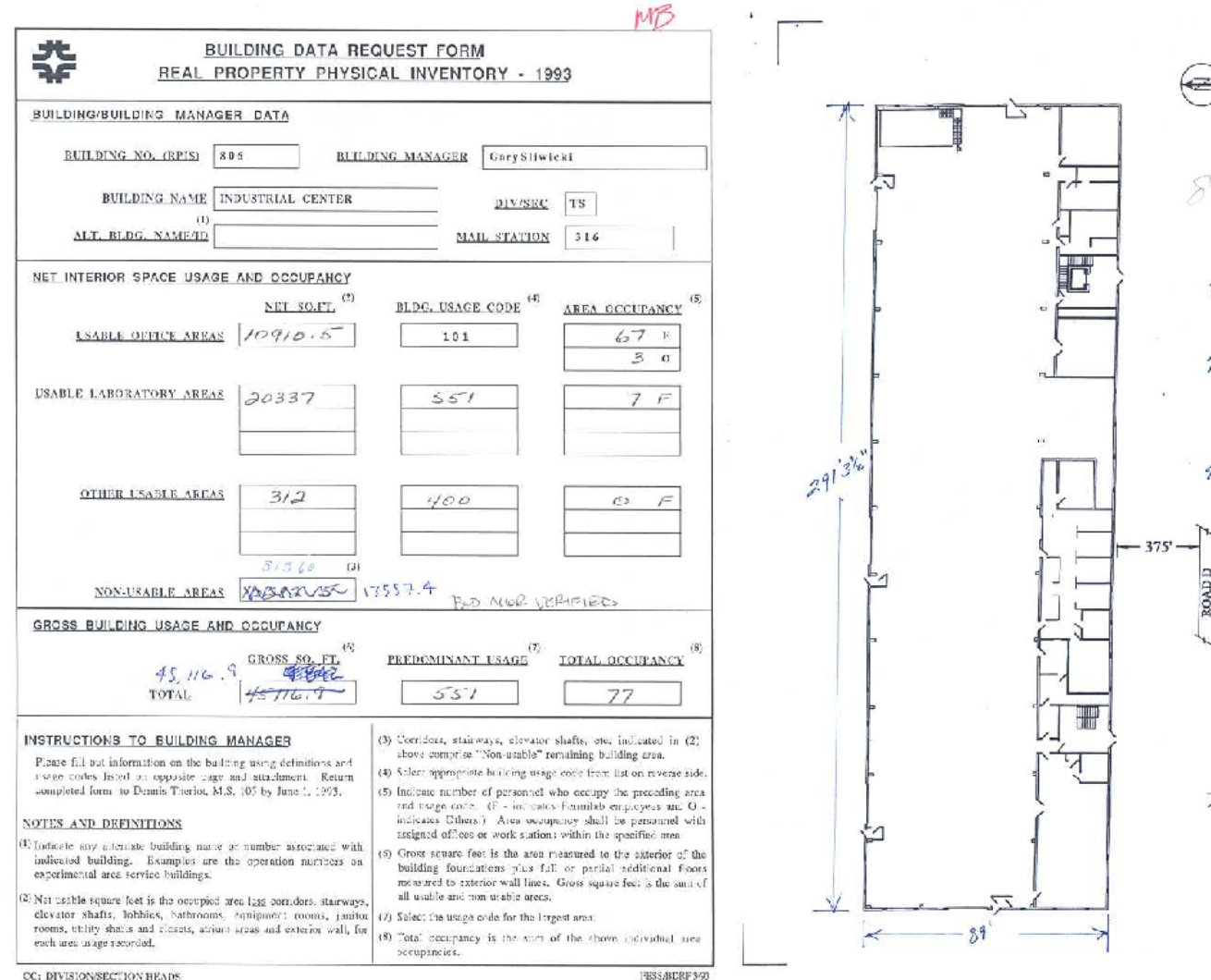

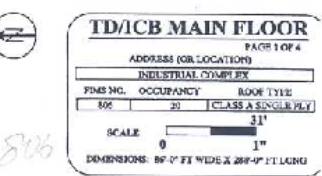

Baserment.

$26^{\circ} \times 96^{\circ}=2,49649.14$

Megg leve?

$26^{\circ} \times 290^{\circ}=7,542,44$

2 leneis $=15,08049$ th

Wlegt on hie bay floor

$2 f^{\prime} \times 20^{\circ}=480 \mathrm{d4}-\mathrm{ft}$.

Budge dim

$291^{\circ} 3 \%^{\prime \prime} \times 89^{\circ}=$

25, $925 \mathrm{ag}, \mathrm{fr}$.

43.981 . 29. 41

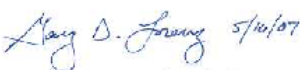

os-sypt dept.

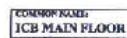

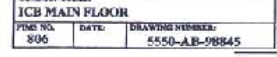




\section{General - Dry}

CONFERENCE ROOM COUNTING ROOM

CROWS NEST

DISPLAY AREA

- SHELTER

- SHOP

- technical area

- THEATER
General - Wet Power - Intensive

- ALCOVE

- BREAKROOM

- CAFÉ

- ClassRoOM

- DINING

- KITCHEN

- lab (ScIENCE)

- LAUNDRY

- MATERIALS PROC AREA

- PIT

- QUIET ROOM

- RECROOM
- COMMUNICATION ROOM

- cOMPUTER ROOM

- LAB (MECHANICAL)

Ventilation - Intensive

- circulation area

- CLEANROOM
High Bay

- HIGH BAY

gARAGE

- LIBRARY

- LOCKER ROOM

- PLATFORM

- STORAGE
Office

- AISLE

- hallway (OFFICE)

- ofFICE

- STORAGE (OFFICE)

\section{Not Usable Area}

- analog room

- ATTIC

- BATHROOM

- BEAM

- BRIDGE

- CATWALK

- CHASE

\section{- CHUTE}

- COLUMN

- CLOSET

- CORRIDOR

- DUMBWAITER

- electrical RoOM

- elevator

- entry (VEstibule)

- EQUIPMENT ROOM
- escalator

- gaRden

- janitor RoOM

- LIVING ROOM

- ENTRY (LOBBY)

- MECHANICAL ROOM

- PARKING

- PORCH
Residential / Not Usable Area Legend Green - Could transform to Usable Purple - Not Usable by FIMS definition Blue - Like Purple; would never be Usable Shaded - "Fermilab' Space Type
- RESTROOM

- ROOFOCCU

- ROOFUNOC

- SHAFT

- STAIRWELL

- tRAVELATOR

- TUNNEL

- UTILITY ROOM

- VAULT
Residential

- BEDROOM

- LIVING ROOM

- DINING ROOM

- OFFICE (HOME) 


\section{Source Documentation - Low Level Waste Handling Building}

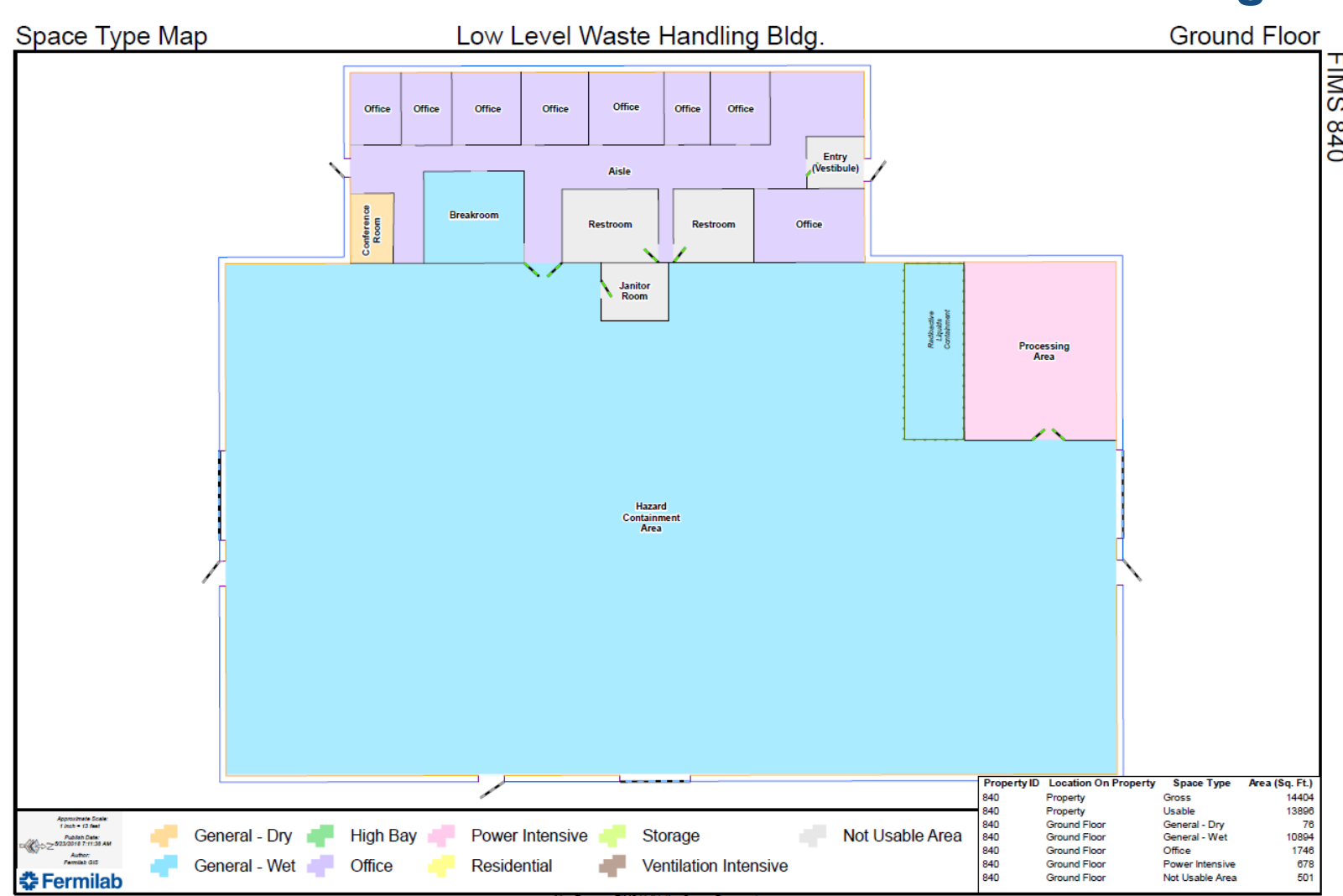




\section{Source Documentation - Fuel Service Center}

Space Type Map

Fuel Service Center

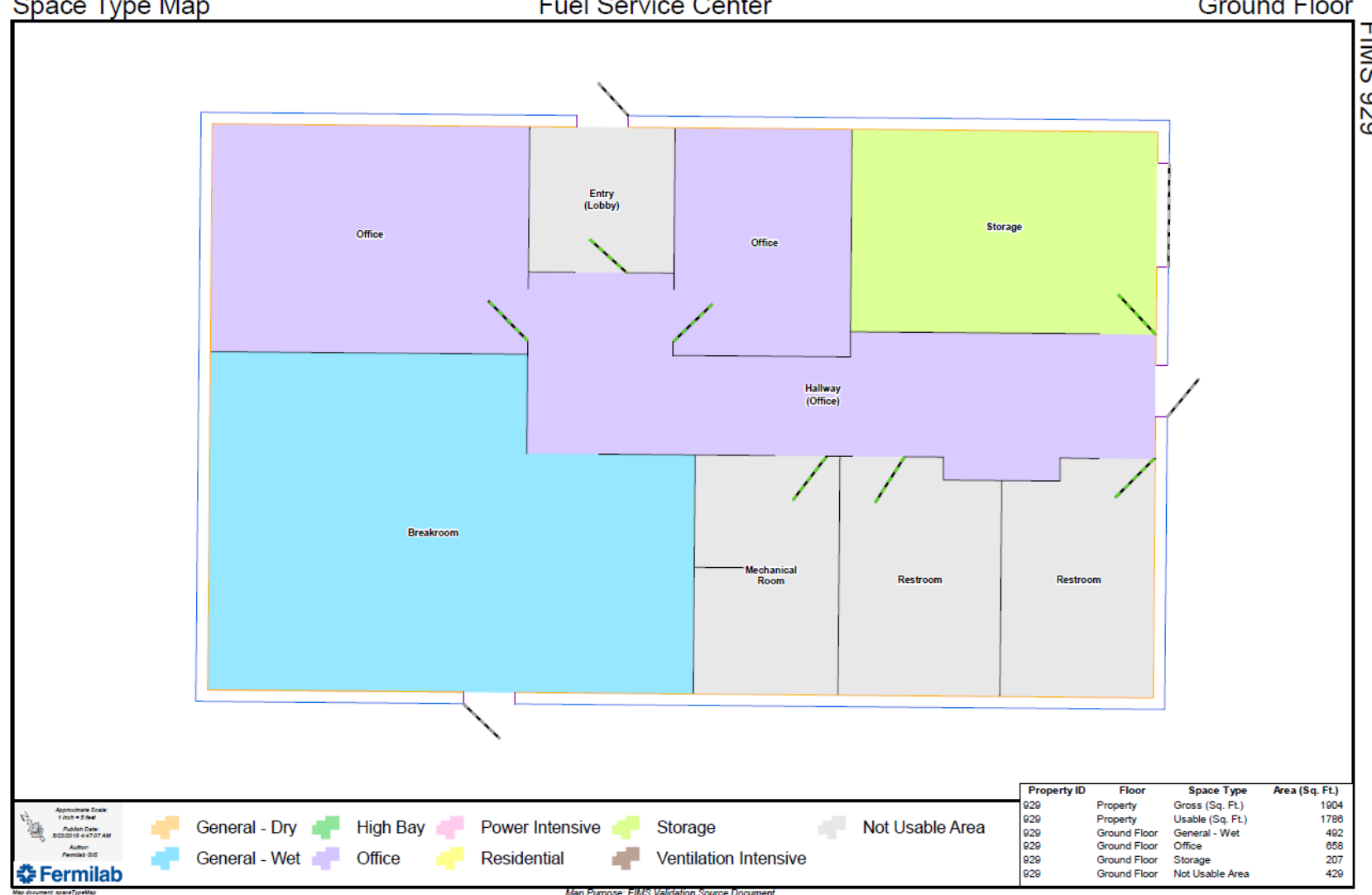




\section{Source Documentation - Science Education Center}

Space Type Map Science Education Center Ground Floor

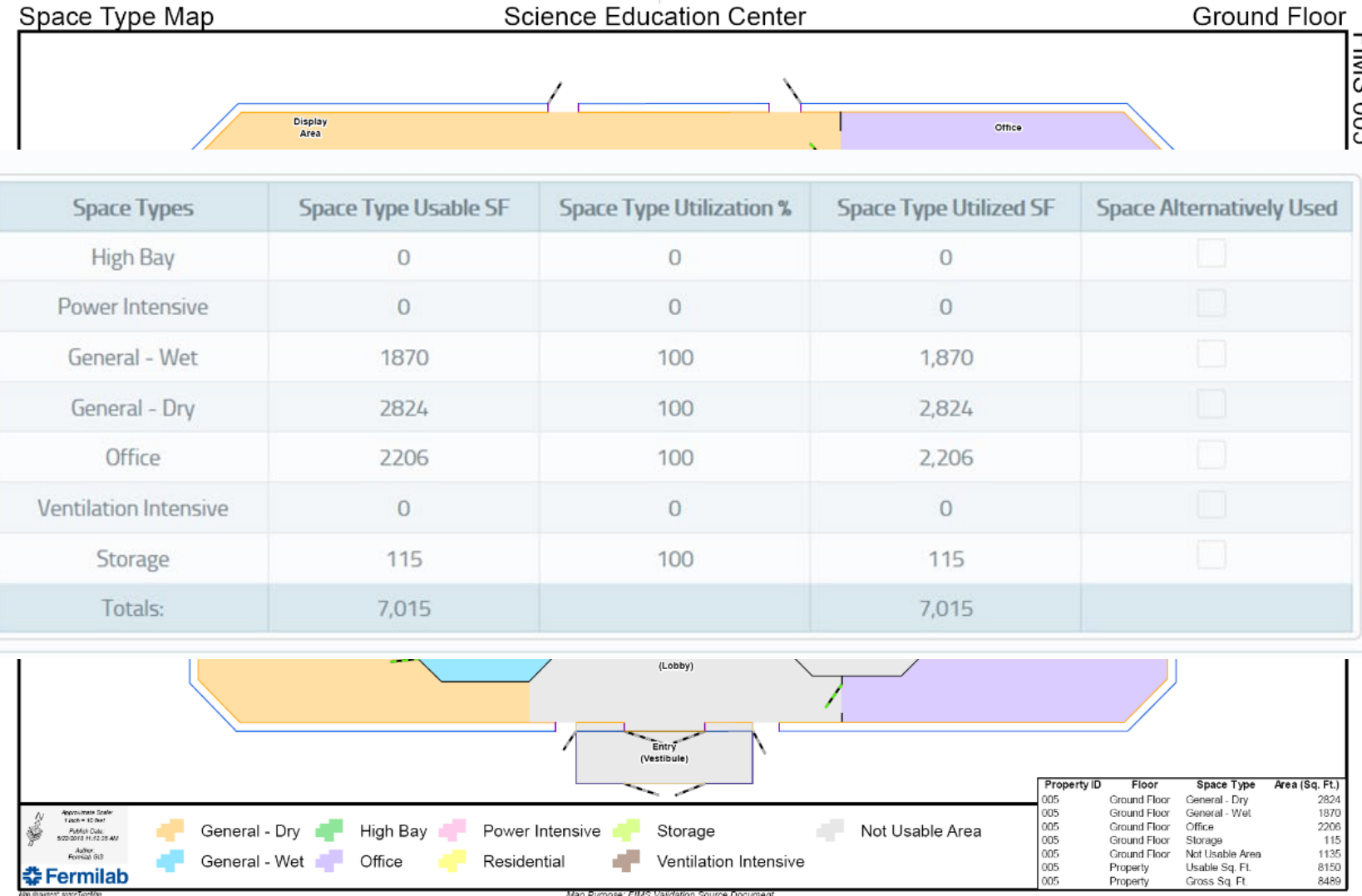




\section{Mobile GIS - Condition Assessments}
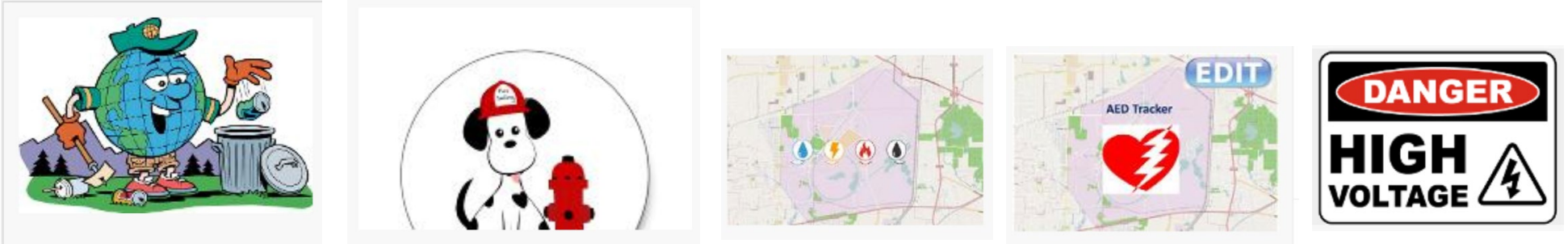

- Using GIS in the field has been valuable for completing in field updates and inventories such as tank locations, guard rails, waste receptacles, sampling locations, utility pole inventory, seed collections, invasive plants, asset tagging

- Mobile GIS applications are used to perform condition assessments for Parking Areas, Cross-Drains to determine asset condition.

- Asset Condition required for annual FIMS reporting - Adequate, Inadequate, Substandard

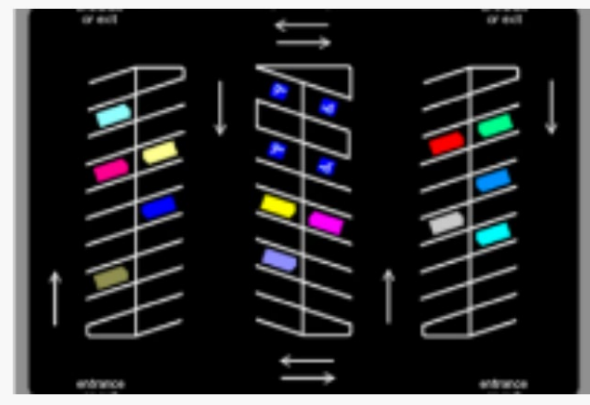




\section{Parking Areas Condition Assessment}

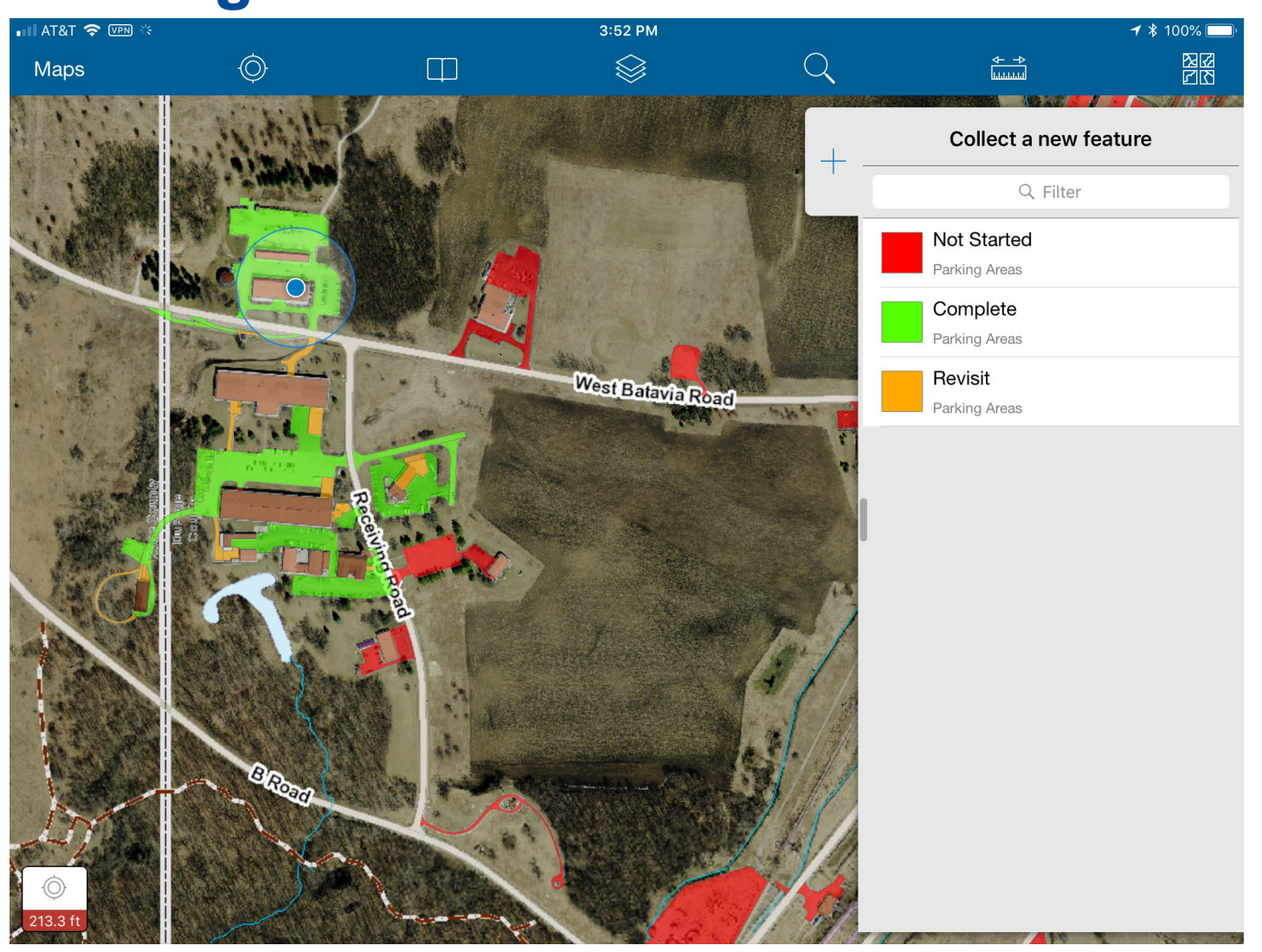

- Assessment is completed in the field

- Collector for ArcGIS (iPad)

- Status of Progress 


\section{Parking Areas Condition Assessment}

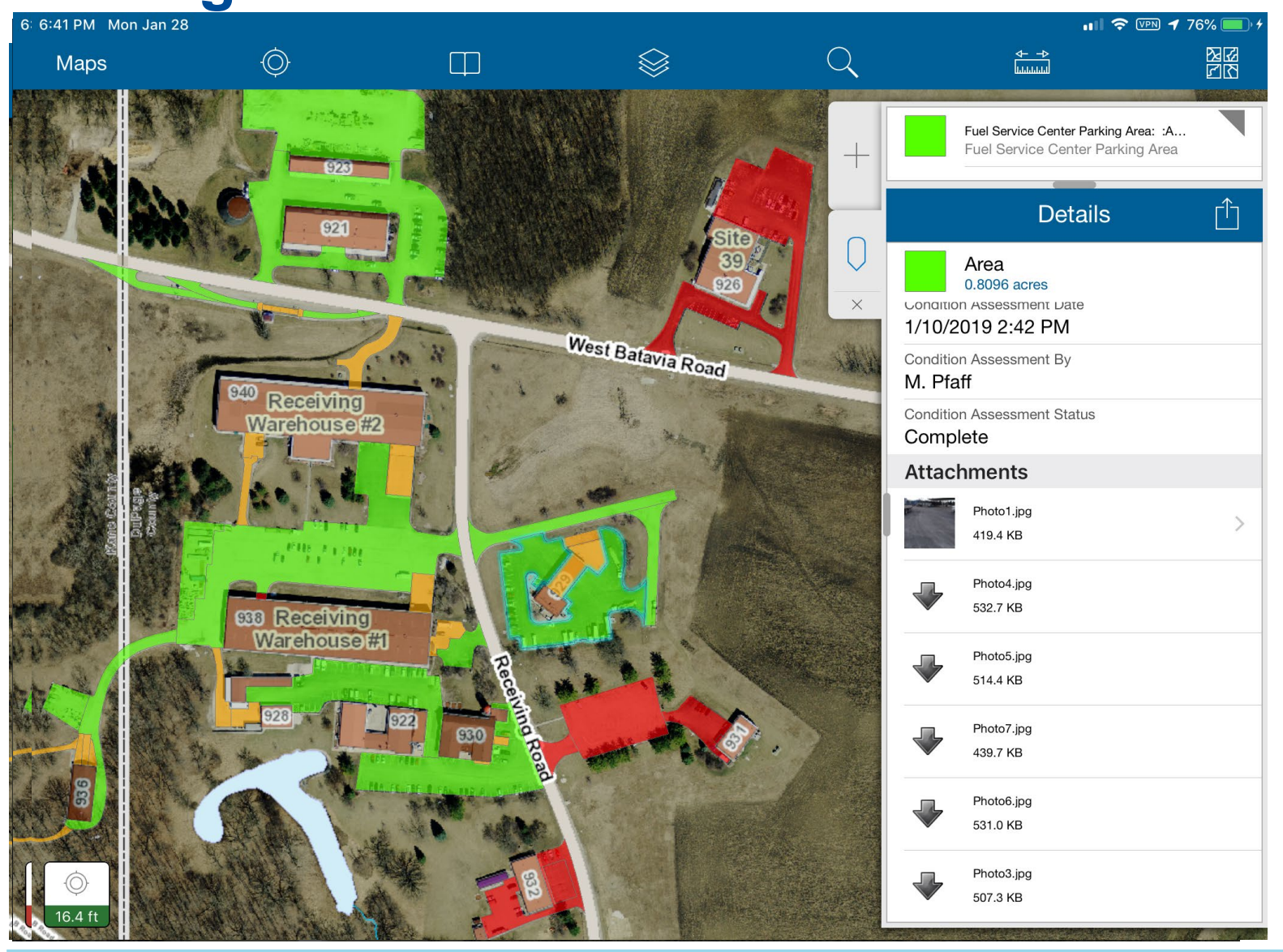

- Condition Assessment includes questions on

- Striping

- Surface

- Base/binder

- Subsurface

- Curbing \& Bumpers

- Date, Name \& Status

- Photos 


\section{Parking Areas Condition Assessment}

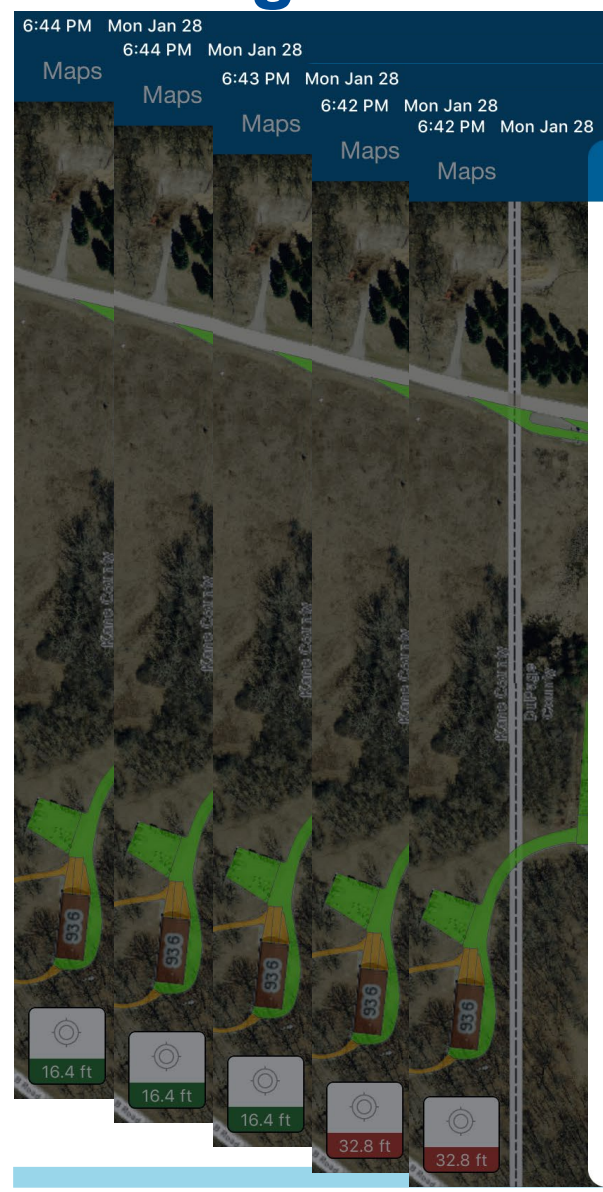

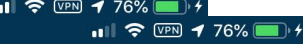

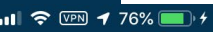

(11) ล $176 \%$ 田 4

- Provide a critical historical record of condition 


\section{Parking Areas Condition Assessment}

- Once complete, the information will be scored to determine an overall Asset Condition.

- Asset Conditions will be migrated to respective condition assessment information data stores and reports.

- The information is also used and analyzed to support budget decisions for work repairs, replacements.

- In field assessments capture accurate, in place information; provide a historical record for a sustainable, repeatable, and maintainable practice. 


\section{What's Next?}

- Fermilab Location Data Standards; rollout and implementation

- Refine, publish and rollout space to space type cross walk standards

- Continue to enhance our FIMS Validation Source Documentation practice

- Continue to enhance and building out GIS integration links to other core systems

- Empower property managers to assess assets; maintain (update, change) assets; condition assessments

- Enhance our capabilities to provide common operating pictures and increase situational awareness across lab functions utilizing our geospatial investments

- Evangelize, collaborate with and continue outreach on our approaches on Location Data Standards and other practices to other DOE Labs 


\section{Thank You}

- Questions

- Comments 